Adams JL, Schonlau M, Escarce J, Kilgore M, Schoenbaum M, Goldman DP. Sampling Patients Within and Across Health Care Providers: Multi-Stage Non-nested Samples in Health Services Research. Health Services and Outcomes Research Methodology. 2003; 4(3): 151-167.

\title{
Sampling Patients Within and Across Health Care Providers: Multi-Stage Non-nested Samples in Health Services Research
}

\author{
John L. Adams, Matthias Schonlau, José J. Escarce, \\ Meredith Kilgore, Michael Schoenbaum, Dana P. Goldman
}

\section{RAND}

\begin{abstract}
In order to better inform study design decisions when sampling patients within and across health care providers we develop a simulation-based approach for designing complex multi-stage samples. The approach explores the tradeoff between competing design goals such as precision of estimates, coverage of the target population and cost.

We elicit a number of sensible candidate designs, evaluate these designs with respect to multiple sampling goals, investigate their tradeoffs, and identify the design that is the best compromise among all goals. This approach recognizes that, in the practice of sampling, precision of the estimates is not the only important goal, and that there are tradeoffs with coverage and cost that should be explicitly considered. One can easily add other goals. We construct a sample frame with all phase III clinical cancer treatment trials that are conducted by cooperative oncology groups of the National Cancer Institute from October 1, 1998 through December 31, 1999. Simulation results for our study suggest sampling a different number of trials and institutions than initially considered.
\end{abstract}


Simulations of different study designs can uncover efficiency gains both in terms of improved precision of the estimates and in terms of improved coverage of the target population. Simulations enable us to explore the tradeoffs between competing sampling goals and to quantify these efficiency gains. This is true even for complex designs where the stages are not strictly nested in one another.

Keywords: Clinical Trials, Sampling Bias, Sampling Studies, Simulation

\section{Introduction}

National probability samples hold great promise for providing unbiased answers to key questions in health services research (Shapiro et al., 1999). However, such approaches are exceedingly rare. In part, this reflects the cost of implementing such a design, but sampling issues also pose a considerable challenge (Frankel et al., 1999). Classic multi-stage sampling methods often cannot accommodate the complex structure of the heath care delivery system.

In classic, multistage sampling, the structures are strictly nested, thereby making them suitable for cluster sampling and stratified sampling schemes. For example, the National Health Interview Survey (NHIS) conducts annual surveys of approximately 40,000 households. First, the NHIS selects counties or groups of contiguous counties as primary sampling units. Then, within these primary units, a sample of blocks (segments) is selected after stratifying by minority population density. Households are then chosen within these sampled blocks.

However, health services researchers often want to construct a sample of patients from various medical groups, for example to examine how patients with a specific disease are treated. Although patients do nest within managed care organizations and possibly within provider groups, they may see multiple primary care physicians and specialists and visit several clinics and hospitals. These complex relationships present design and sampling as well as analytical 
challenges. Frequently these complex structures are forced into nested designs even though they are inherently not nested.

This paper introduces a sampling methodology for multi-stage sampling of health service users. The approach described here can be applied to any complex sampling problem where the levels of the structure are not strictly nested. We consider all design alternatives that are thought to be of interest, including sampling with equal probability and proportional to size as special cases. Further, we explore alternative definitions of size. We consider different design goals and use simulations to evaluate them and make the tradeoff between the competing goals explicit.

\section{Background}

The primary goals for study design should be to maximize the analytical information while controlling data collection costs. The example above is instructive. The naïve approach might be to sample patients at random and then go to all of their providers to review medical records for patient characteristics and care received. This approach can result in "snowballing" data collection costs as abstracters go from office to office to find the required charts. To keep costs down, the design should capitalize on the lower marginal costs of data collection at providers already being visited. Care must be taken, however, to ensure that the sample does not end up concentrated only in the high volume providers or over-clustered in a way that hurts the effective sample size.

A similar situation arises in other health services research contexts. The Cost of Cancer Treatment Study (CCTS) was designed to measure the incremental costs of care in cancer clinical trials (Goldman et al., 2000). The issue of how to pay for clinical care for patients in cancer trials has received much attention recently. Most insurers or plans have policies that exclude coverage for services given as part of a clinical trial (Mansour, 1994, Morrow et al. 
1994, Wagner et al., 1999). The CCTS was conceived to provide precise, generalizable estimates of the cost of conducting government-sponsored cancer trials.

To reach patients who are enrolled in clinical trials for cancer, it needed to address two levels of organizational structure: clinical trials and institutions. Institutions are not strictly nested within clinical trials, because many institutions participate in more than one trial. Most trials are multisite and not nested within institution. An important feature of this problem is that the cost of data collection is dominated by the fixed costs of adding another trial or institution to the study, rather than the variable cost of adding patients within a trial-institution pair. Classical, multi-stage sampling suggests three potential sampling schemes, each with its own problems.

A typical multi-stage sampling scheme would first sample trials proportional to size, and then select institutions at which a trial is conducted with equal probability in a second stage. (Kish, 1965, Chapter 7). We assume a complete listing of trial-institution pairs. Variations on this approach include sampling with equal probability in both stages, or equal probability in the first stage and proportional to size at the second stage (Kish, 1965). However, these approaches ignore that it is more economical to sample an institution that has already been sampled for another trial than one that has not been sampled yet. Consequently, typical designs are likely to sample more institutions than would be cost efficient.

Another approach might be to list all possible pairs of trial-institution pairs and to sample with equal probability or proportional to size. This approach also ignores potential economies and will result in a sample that is spread across too many trial-institution pairs. A third possibility might be to sample a fixed number of trials with equal probability or proportional to size and sample all institutions at which these trials are conducted. If total costs are fixed, this is 
likely to result in a design that has too few trials relative to the number of institutions sampled. This is inefficient compared to alternative designs.

As our examples suggest, complex designs often have different competing goals. Statisticians consider small design effects (the design effect specifies the loss or the gain in precision of a design relative to simple random sampling), low or fixed cost, and small frame error (e.g., due to ignoring very small trials and institutions) of the study as worthy design goals. Sampling proportional to size might best support one goal of the study, whereas another goal might be best supported by equal probability sampling.

Classical texts (Cochran, 1997) emphasize that it is important that the sampling frame cover the target population well, but then focus on the tradeoff between cost and precision. The tradeoff between precision and coverage (sampling units with non-zero chance of being sampled) can be viewed as a tradeoff between variance and bias. This tradeoff is not usually explicitly considered. Instead one usually focuses on the mean squared error, which specifies one specific tradeoff between bias and variance. The "Total Survey Design” approach (Anderson et al., 1979, Linacre et al., 1993) aims to design surveys such that the total error, a generalization of the mean squared error, is minimized given a fixed cost constraint. For this approach to work, various bias components, including the one stemming from a lack of coverage, need to be modeled explicitly. This requires either additional auxiliary data sources or can be accomplished by conducting an evaluation survey prior to the actual survey. The mean squared error of a variable of interest $y$ is defined as

$$
\operatorname{MSE}(y)=\operatorname{Var}(y)+\operatorname{Bias}^{2}(y)
$$

In our case the variance term is the variance of the quantity of interest in the covered portion of the sampling frame. The bias term is a measure of how different the quantity of 
interest is in the uncovered portion of the sampling frame. In the absence of data or other information on how the coverage affects bias the bias term is ill defined Instead, we specify the coverage as a percentage of the target population to establish a bound. Precision and coverage bias are combined through the use of the generalized mean squared error in the "Total Survey Design” approach. We enable the user to explicitly examine the tradeoff to make an informed decision.

\section{Method}

Our approach to picking a sampling design is the blueprint for our plans to develop a more general sampling design development tool. The four steps are summarized in Figure 1.

** Figure 1 approximately here ***

We now explain these steps in detail, using the Cost of Cancer Treatment study to illustrate the concepts. For the CCTS we obtain a random sample of cancer patients enrolled in clinical trials. Patients are enrolled in clinical trials through a medical institution. We employ a two stage sampling design, in which we first sample clinical trials and then sample institutions involved in the sampled trials. We use all patients who enrolled in any of the sampled trialinstitution pairs. (In other settings a third stage of sampling patients within trial-institution pairs might be used.) For each trial-institution pair we also select a control group of the same size with the same cancer from the non-trial participants. The control group is drawn within the same institutions as the trial participants. Finally, we remove any trials or institutions that do not enroll a pre-specified minimum number of patients. This final step makes the design more cost efficient. It is also the reason why the design is not strictly hierarchical. This approach is useful when the constraints on the design space are complex or when sampling one unit can have a substantial effect on the cost of sampling another unit. 
This approach is not restricted to a two stage sampling design with restrictions on the minimum number of sampling units. This problem is merely an example of how to implement our approach.

\subsection{List a set of sensible sampling designs (usually parameterized)}

Having a choice among several sampling designs is always better than considering just one sample design. We consider several designs and then choose the design that yields the best compromise among all goals of a particular study. To elicit a candidate list of sensible designs, it is helpful to identify design parameters.

For example, one might consider designs that give larger clusters a higher probability of being sampled versus designs that assign equal probabilities regardless of institution or trial size. For the CCTS this means giving larger trials or larger institutions a higher probability of being sampled. These choices can be conveniently quantified through one design parameter which we label $\alpha$. We select clinical trials in the first stage and institutions in the second stage proportional to their size raised to the power of $\alpha$. (Different values for $\alpha$ could be employed in different stages if desired). The size of a clinical trial can refer to the cumulative number of patients who enrolled in the trial over a specified period of time. The size of an institution refers to the number of patients enrolled in any of the clinical trials sampled in the first stage. This means that the definition of size for an institution will change with the trials selected in the first stage. The parameter $\alpha$ determines whether sampling units are selected with equal probability $(\alpha=0)$, proportional to size $(\alpha=1)$, or any intermediate function of size. This parameter allows us to compromise between a simple random sample and a sample that takes more large units. We restrict $\alpha$ between 0 and 1 . For simplicity we use the same value of $\alpha$ for selecting both clinical trials and institutions, although it is not necessary to restrict the $\alpha$ to be the same for both stages. 
Coverage is solely a function of the two cutoffs. Neither $\alpha_{1}$ nor $\alpha_{2}$ affect the coverage; they only affect the distribution of the sizes of the trials and institutions in the sample (above the cutoff points). Both coverage and $\alpha$ are input parameters. Because we chose a factorial design, in our example coverage and $\alpha$ are deliberately uncorrelated. The same reasoning applies if we chose different alphas in both stages. Bias is considered only as a function of coverage.

Because the per-patient cost of sampling institutions and trials with few patients is very high, we investigate the effect of only sampling institutions and trials with a specified minimum number of patients. Thus the two remaining design parameters that we use in this study are the "trial cutoff," the minimum number of patients that a trial must have accrued in order for it to be eligible for sampling in the first stage; and the "institution cutoff," the minimum number of patients accrued in sampled trials at an institution in order for it to be eligible for sampling in the second stage. Once a sample of trials is selected we construct a list of all institutions that accrue patients in one or more of these trials. We then eliminate all institutions from this list in this particular sample that have not accrued the minimum number of patients across all trials sampled in this particular sample. Note that which institutions reach this cutoff value depends on which trials are sampled at the first stage. Small institutions which are costly to sample on a per patient basis are less likely to be sampled; but when sampled they have higher weights in order to represent the population of small institutions. In terms of design goals, these two cutoffs determine the tradeoff between coverage and the precision of the estimates. While these two parameters are specific to the class of design problems for which small clusters are undesirable, they exemplify how to parameterize awkward design problems.

All parameters mentioned so far are tuning parameters. They are not directly subject to economic constraints in that they only shift sample to different places in the design space. By 
contrast, the number of units to sample at each sampling stage are subject to a cost constraint. Typically, the number of sampling units that we can afford to include in the study at each stage (trials and institutions for the CCTS) has been established in the proposal writing process. As the fixed cost of sampling trials and institutions may be different, we specify a list of equal cost alternatives. We can sample more sampling units in the first stage at the expense of less sample units in the second stage while the overall cost remains roughly constant. The design process selects from this list while simultaneously considering the other parameters.

The number of trials and institutions sampled also limit the sample size and often a minimum sample size is needed to ensure estimates that are sufficiently precise. Early on in the design process rough power calculations are useful to determine the sample size needed, which can then be translated to the number of institutions and trials required to attain the sample size.

\subsection{Estimate sampling probabilities via simulations}

The purpose of the simulations is to estimate the sampling probabilities $p_{i j}$ where $i$ indexes trials and $j$ institutions ( i.e., the sampling probabilities of trial-institution pairs) which are needed to compute the design effects. In complex designs, sampling probabilities are difficult or impossible to compute analytically. Even study designs that initially seem straightforward may become more elaborate as the study develops. A simulation-based approach maintains flexibility to accommodate unforeseen challenges that may only surface in a later stage of the study design. Complex designs arise, for example, when there are non-trivial constraints on the design space, or as in the CCTS study, when the design is not strictly hierarchical. For the CCTS study, we simulate the probabilities of sampling institution-trial pairs by drawing a "large number" of two stage samples. We choose a number large enough to estimate the coverage correctly, meaning each institution/trial pair that has a positive selection probability is sampled at 
least once. We accomplish that by choosing the "large number" such that each institution/trial pair that is sampled is sampled not only once but at least 5 times. For the CCTS study this number turns out to be 7500. The rationale is as follows: the chance that we never sample an institution/trial pair that has the same probability as the smallest probability that we do sample is $(1-5 / 7500)^{7500}=0.00672$. In other words, it is very unlikely that we miss such an institution/trial combination. Of course, 5 is an arbitrary number and can be changed.

We then estimate the sampling probability for each trial-institution pair by the fraction of times it appears in these samples. We denote the probability with which a trial-institution combination is selected by $p_{i j}$. Because we sample all patients within a sampled trial-institution pair the probability of sampling a patient is simply equal to the probability of sampling that trialinstitution pair. In other studies it may be advantageous to subsample patients or to sample patients with different characteristics with different probabilities. For example, the study may wish to over sample non-white patients to achieve a minimum effective sample size for subset analysis. This is easily accommodated in this approach by adding another probability to the product of the two stage probabilities corresponding to the probability of sampling a patient conditional on having sampled the unit in which they reside.

A single two-stage sample consisting of $I$ clinical trials and $J$ institutions is constructed as follows. We first sample $I$ clinical trials that meet the trial cutoff proportional to their size raised to the power of $\alpha$. Then a list of all institutions participating in any of the $I$ clinical trials is constructed. We remove all institutions that do not accumulate enough patients across the sampled trials to meet the institution cutoff. From that list we draw $J$ institutions proportional to the size of the institution raised to the power of $\alpha$. All sampled institutions contribute their 
patients participating in the trials that were sampled at the first stage. A sampled trial-institution pair is any sampled trial combined with any of the sampled institutions participating in that trial.

\subsection{Evaluate the draws with respect to multiple goals}

In evaluating a survey design, we consider the goals of high precision of estimates and good coverage meeting the cost constraint. It is very difficult to give an explicit formula of how cost depends on institutions and trials. However, when the numbers of institutions and trials are the same for a set of design choices, the cost is roughly the same across this set. We investigate three scenarios within which the sum of the number of institutions and trials remains constant. Efficiency gains can then be given relative to another scenario: "we can increase the effective sample size by $\mathrm{x} \%$ if we sample y more institutions and $\mathrm{z}$ less trials." (Sample size refers to the number of patients, not the number of trials or institutions. A definition of effective sample size is given later).

Coverage refers to how well the sampling frame represents the target population. The target population for the CCTS study consists of all patients in phase III clinical cancer trials that are conducted by cooperative oncology groups of the NCI between October 1, 1998 and December 31, 1999. For the CCTS the sample frame equals the target population. Because this is not usually the case we express coverage relative to the sample frame. That is, a $100 \%$ (50\%) coverage means that all (half of the) patients in the sampling frame have a nonzero chance of being selected. A nonzero institution cutoff deletes either small institutions or institutions whose patients are scattered across many trials. The coverage depends on the two cutoff values in a non-obvious way. It is determined by dividing the number of patients that were selected at least once during the simulation by the number of patients across all trial / institutions. 
The statistical efficiency in estimating the parameters for the CCTS study is not as good as the efficiency for random sampling because the sampling probabilities are unequal. Unequal sampling probabilities induce unequal weights which leads to larger standard errors. It is common to evaluate precision based on the effective sample size, $n_{\mathrm{eff}}$, rather than looking directly at the standard errors. The effective sample size depends on the particular estimator; here as an example we use the effective sample size for the weighted mean. The somewhat more complicated design effect we actually used for the CCTS study is discussed in the appendix. The effective sample size $n_{\text {eff }}$ is a function of two quantities:

$$
n_{\mathrm{eff}}=n / D E
$$

where $n$ is the actual sample size and $D E$ the design effect. The effective sample size can be interpreted as the size of a simple random sample yielding the same precision. Simple random sampling can be viewed as a sampling strategy with a design effect of 1 .

Assuming a block diagonal variance/covariance matrix the overall design effect (Gabler et al., 1999) is the product of the probability design effect $D E_{p}$. and the cluster design effect $D E_{\rho}$ :

$$
D E=D E_{p} * D E_{\rho}
$$

The probability design effect quantifies the loss of efficiency that we induce by not sampling with equal probability. The probability design effect, $D E_{p}$, can be computed as

$$
D E_{p}=\frac{n \sum_{i} \sum_{j}\left(n_{i j} w_{i j}^{2}\right)}{\left(\sum_{i} \sum_{j} n_{i j} w_{i j}\right)^{2}}
$$

where $w_{i j}$ is the weight for the trial $i$-institution $j$ pair, and $n_{i j}$ is the number of patients accrued there. The indices refer to trial-institution pairs in the sample, not to all possible trial-institution pairs. The weights are given by $w_{i j}=1 / p_{i j}$, where the probabilities $p_{i j}$ were estimated in the first 
simulation as described earlier. Sampling with equal probability yields a probability design effect of 1 .

The cluster design effect quantifies the loss of efficiency by sampling clusters rather than individual elements. Measurements of elements in clusters tend to be more correlated than those of random elements. For computing the average cost the cluster design effect, $D E_{\rho}$, is

$$
D E_{\rho}=1+\rho(B-1)
$$

where B is the average cluster size and $\rho$ the intra-cluster correlation coefficient. As equation (2) shows, the loss of efficiency - or design effect - increases with stronger intra-cluster correlation and greater average cluster size.

When computing the difference in costs between cases and controls rather than the average cost it is not obvious what the cluster design effect is. Controls are cancer patients, comparable to cases, who received care at the participating institutions but did not enroll in any clinical trial. We derive the design effect in Appendix A assuming that observations within trial/institution pairs are correlated and the between pairs correlation is negligible. We believe this is a reasonable assumption in the design stage for many problems. In the design stage, the largest correlation is often used to approximate the complete correlation structure. In this case it is wise to assume a somewhat larger value for the correlation. If the study designer has intuition or data on several correlations they could easily be incorporated using a derivation analogous to the derivation in Appendix A. In the CCTS example we have assumed that the dominant correlation is the within trials correlation. If our candidate designs were found to select many trial / institution pairs from single institutions we would need to reconsider the importance of the within institution correlation. 
We draw a large number of two-stage samples and compute the design effect and the sample size for each sample. This yields an empirical joint distribution for the actual sample size, the design effect, and the effective sample size. However, the distribution is generated by drawing many samples from a particular study design. For any one study like the CCTS we draw only one sample, and it is important to also investigate how bad a single unlucky draw may be. Unlucky draws correspond to the upper tail of the distribution of the design effect. Hence a good distribution has two features: the average (or median) design effect is small, and the distribution of design effects does not have a large right tail. We therefore monitor both the median and the $90^{\text {th }}$ percentile of the distribution of the design effect. Generally, the design effect tends to decrease the more homogeneous the selection probabilities, $p_{i j}$, are. Excluding trials and institutions with small accrual, i.e., raising the cutoffs, tends to accomplish that.

\subsection{Choose the design that is the best compromise among the goals}

As mentioned before, we consider the goals of high precision of estimates and good coverage meeting the cost constraints. Usually all design goals cannot be optimized simultaneously, therefore, the design of choice is a compromise. If the compromise could be quantified in form of a loss or utility function, one could engage in formal optimization of the loss function. In practice the researcher rarely has an explicit tradeoff between competing design goals in mind. Also, routine optimization may overlook attractive alternatives. We prefer a conscious compromise in which we are aware of the tradeoffs. What tradeoffs are acceptable very much depends on the context of the problem at hand. Once a design has been chosen, a single final sample is drawn.

The method described here has been implemented in Stata. The program can be obtained by sending an e-mail to dana_goldman@rand.org. 


\section{Results}

The goal of the Cost of Cancer Treatment Study (CCTS) is to estimate medical costs incurred by patients who enroll in a clinical trial relative to patients who do not enroll in any clinical trial. The target population for the CCTS study consists of all patients in phase III clinical cancer trials that are conducted by cooperative oncology groups of the NCI. The data were collected between October 1, 1998 and December 31, 1999.

The original power calculations were a typical lognormal health care costs analysis. We assumed that health care costs are positively skewed and are normally distributed after a logarithmic transformation. Similar data was analyzed and an unadjusted coefficient of variation of 0.95 was observed. In similar settings we have typically achieved reductions in variance of costs of $15-20 \%$ through multivariate analysis. We assumed the same coefficient of variation for both trial participants and the non-trial participant control groups. With these assumptions 750 cases and 750 controls would have $80 \%-90 \%$ power to detect a cost difference of $10 \%$.

The power calculations are only used to guide a first choice of the number of trials and institutions needed. Guidance as to which values to choose for the trial and institution cutoffs can be obtained from looking at histograms or simple frequency tables of institution and trial sizes. Figure 2 shows how many patients were accrued at trials by size of the trials for the CCTS study. Figure 3 shows the analogous histogram for institutions. A small number of simulations with very few iterations can also help to quickly distinguish between realistic and unrealistic parameter values.

\footnotetext{
*** Figures 2 and 3 here $* * *$
} 
Based on these histograms and on initial simulations, we consider trial cutoff values of 18,24 , and 30 , and we consider institution cutoff values of 6,9 and 12 . There is no hard rule as to how to choose these numbers. It is important, however, to examine a wide range of cutoffs.

For the trial and institution count values we consider three scenarios: sampling 35 trials/ 55 institutions; 50 trials/ 40 institutions and 45 trials/ 50 institutions. For each scenario, we then consider 3 different values for each design parameter: 18, 24, and 30, for trial cutoff; 6, 9, and 12 for institution cutoff; and $0.5,0.75$ and 1 for $\alpha$. This yields $3 * 3 * 3 * 3=81$ possible combinations of design parameters. For each of the 81 runs, the simulations are as follows: For each simulation, we run 7500 iterations to determine the sampling probabilities.

For each simulation, we select the 5 largest institutions purposively and the remaining institutions as described earlier. We would likely reject any sample that does not include many of the very large institutions. Rather than rejecting undesirable samples and making sampling probabilities unnecessarily difficult to compute, we purposively select some institutions and let the corresponding smaller weights reflect this inclusion. After selecting the trials we set the inclusion probability of some institutions to one and simulate the inclusion probabilities of the remaining institutions. Note that the largest institutions vary from sample to sample depending on the trials selected.

Purposively including some institutions (i.e., sampling some institutions with probability 1) does not constitute "cheating." On the contrary, it illustrates how easy it is to accommodate study specific inclusion criteria by making minor modifications to the simulations. Not doing so would mean modeling a slightly different sampling approach than is actually used. The effect of incorporating this common practice in our model is a slightly larger design effect. 
Each sample for the simulation in Step 2 can be drawn as follows: (a) A list of all trials is constructed. Trials with too few patients according to the trial cutoff are eliminated from this list. (This only need be done once for each design). (b) A sample of trials is drawn from this list. (c) From the sampled trials a list of associated institutions is constructed. Institutions that do not have enough patients based on the institution cutoff are eliminated from this list. (d) Institutions are sampled from this list. The sample consists of all institutions/trial pairs for which both the trial and the institution were sampled.

Having determined the sampling probabilities we were then able to estimate the design effect. We ran 400 additional iterations and computed the design effect for each of the iterations using equation (1). The calculation of the design effect requires the sampling probabilities $p_{i j}$, which can only be computed after the last run of the first simulation.

In the Stata implementation of the program a simulation that draws 7500 samples to compute the probabilities $\mathrm{p}_{\mathrm{ij}}$ and 400 samples to compute the design effect based on the $\mathrm{p}_{\mathrm{ij}}{ }^{\prime} \mathrm{s}$ takes about 1.6 hours on a laptop with a $1 \mathrm{Ghz}$ processor and 512K of RAM. Simulations can be run in parallel. For example, with 4 workstations it is possible to run about 32-36 simulations over night.

As a first step in selecting the design, we can now choose between the three trial and institution count values scenarios. The curves that show the trade-off between coverage and effective sample size are a useful graphical summary of the three scenarios. Figure 4 presents the tradeoff curves for these three scenarios. For each scenario the 27 design combinations are plotted. To make it easier to distinguish different sets of admissible points for each scenario we connect the admissible points. Admissible points are points for which no other point exists that has both better coverage and a greater effective sample size. The 45 trials/ 50 institutions curve 
dominates the 35 trials/ 55 institutions curve (except in one small section), which in turn dominates the 50 trials/ 40 institutions curve. One curve dominates another if the two curves do not cross. This implies that for any point on the dominated curve there exists a point on the dominating curve that is uniformly better.

We can see clearly as coverage increases effective sample size decreases. As coverage increases the range of the selection probabilities increases, inducing a larger design effect, which translates to a smaller effective sample size.

Our methodology was developed in response to the complexity of this study, but not in time to be used in the design phase of this study. The scenario chosen in our study was 35 trials and 55 institutions. Relative to this scenario we see that adding 10 additional trials and removing 5 institutions is beneficial. However, adding 15 additional trials and removing 15 institutions is a bad idea. Adding 15 additional trials in our study meant sampling almost all (in some cases all) trials eligible that had at least as many patients as specified in the trial cutoff value. This suggests that the turning point at which adding trials at the expense of institutions becomes disadvantageous is reached only after a large fraction of trials in the sample frame is already sampled.

*** Figure 4 here $* * *$

The run corresponding to our study is plotted with a diamond symbol in Figure 4. It has an effective sample size of 793 and a sample frame coverage of 63.37\%. It corresponds to an institution cutoff of 9 , a trial cutoff of 18 , and an $\alpha$ of 1 . This means that we are drawing trials and institutions proportional to size.

Table 1 gives various statistics for all combinations of levels for the scenario with 35 trials and 55 institutions. Specifically, we report on the sample frame coverage, the median 
accrual (i.e., median sample size), the median design effect, the median effective sample size, and the $90^{\text {th }}$ percentile of the design effect. We also report on the median probability and cluster design effects separately. Most of the design effect stems from the unequal weights inducing the probability design effect, relatively little from the clustering. The sample size in Table 1 refers only to the number of cases. Because of the differencing between cases and controls, for the CCTS we obtained an equal number of controls. Our study has the parameters Trial Cutoff $=18$, Institution Cutoff $=9$ and $\alpha=1$. The correlation between the median design effect and the $90^{\text {th }}$ percentile for Table 1 is $42 \%$. Because there is only moderate correlation the run with the best median design effect may not have a favorable $90^{\text {th }}$ percentile design effect.

*** Table 1 here ***

On closer examination, the runs in Figure 4 appear to be clustered in groups of three with each of these groups having the same coverage. The clusters correspond to the three different levels of $\alpha$. This implies, while the choice of $\alpha$ clearly affects the effective sample size, $\alpha$ is less important than the other factors we are investigating (trial cutoff, institution cutoff, number of trials, number of institutions). Moreover, as can be seen from Table 1, all other factors being constant, sampling proportional to size ( $\alpha=1)$ is often inferior to $\alpha=0.5$ and $\alpha=0.75$.

\subsection{Study sample}

Finally, we generate one sample sampling proportionally to size and with cutoffs 18 and 9 for trials and institutions, respectively. The final sample yields a design effect of 1.28, below the median as indicated in the first row of Table 1. After dropping institutions with an accrual of less than 9 patients across the 35 sampled trials, there are about 110 institutions left, 55 of which we sample. Each of the 55 institutions we sample participates in 3 to 14 of the sampled trials. 


\section{Discussion}

We have presented a simulation-based approach for examining multiple sampling goals for a multi-stage study design in which the levels are not strictly nested within one another. In evaluating the sampling goals, we use simulations to estimate quantities such as the sampling probabilities, which cannot easily be computed analytically due to the complexity of the design. In the CCTS we have focused on sample frame coverage and the effective sample size (which is equivalent to considering the standard error of the estimates) and considered cost indirectly by evaluating the implications of trading additional trials for less institutions.

The potential significance of our research is in its contribution to more economical study design. The analysis needs to take into account the design structure. Mixed model regression could be used, for example, specifying trial and institutions as random effects with a suitable covariance matrix. Another option is generalized estimating equations (GEE), which is asymptotically robust against misspecification of the covariance matrix. For the CCTS this option is preferable given the large number of trials and institutions.

The design economies are twofold. First, it is possible to produce sampling plans that may produce more of everything in some cases. The removal of the constraint that the researcher must use one of a relatively small collection of standard approaches can yield better designs for all of the objectives simultaneously in some cases. For example, we have demonstrated that a tradeoff between the number of trials and institutions sampled can lead to tradeoff curves that dominate one another. Second, these methods allow the design team to consider more options more thoroughly. We believe that good tools that help in the design process will prove to be very useful to the researcher. 
Another useful feature of this approach is that it can be easily adapted to problems that arise in the field. For example, samples must occasionally be reduced because of cost overruns; an unexpectedly large number of trials or institutions may refuse to participate or additional funds may make sample expansion possible. Dealing with all these problems while still maintaining a probability sample is straightforward. The simulations are merely expanded to include the additional actions being considered and new sampling probabilities, design effects and coverages are calculated.

Our approach is less attractive when the stages of the multi-stage design are fully nested within one another. In this case the sampling probabilities can be easily computed and need not be simulated. Nonetheless, exploring the tradeoffs among the design goals remains an attractive feature.

\section{Appendix}

In this appendix we derive the design effect for computing the difference between the average cost for cases and the average cost for controls.

Denote the $\log$ costs of cases by $y_{i j k}$, and the $\log \operatorname{cost}$ of controls by $z_{i j k}$. (The logarithm of cost is used so that we can assume a normal distribution and a constant variance in the power calculations below.) As before $i$ and $j$ enumerate the trials and institutions respectively. We assume that the design aims to obtain the same number of controls and cases within each trial/institution pair. We assume the following model:

$$
Q=\frac{\sum_{i j} w_{i j} n_{i j}\left(\bar{y}_{i j}-\bar{z}_{i j}\right)}{\sum_{i j} w_{i j} n_{i j}}
$$


where $w_{i j}$ are the sampling weights and $n_{i j}$ are the number of cases in trial/institution pair $i j$. If there is no difference in cost between cases and controls then $E(Q)=0$. Further, we assume

$$
\begin{aligned}
& \operatorname{Var}\left(y_{i j k}\right)=\sigma^{2} \\
& \operatorname{Cov}\left(y_{i j k}, y_{i^{\prime} j^{\prime} k^{\prime}}\right)=\left\{\begin{array}{ccr}
\rho \sigma^{2} & i=i^{\prime} ; j=j^{\prime} \\
0 & \text { if } & \text { otherwise }
\end{array}\right.
\end{aligned}
$$

where $k$ enumerates the cases within trial/institution pairs. We use the same variance /covariance model for the controls and cases. This is the same variance /covariance structure used by Gable et al. (1999) in justifying Kish’s formula for design effects. In other words, observations within trial/institution pairs are correlated, observations between trials or between institutions are uncorrelated. Other correlations could be incorporated by modifying (3). The correlation structure we specify reflects our belief that the within trial/institution correlation is likely the largest correlation. Assuming $\sigma_{\mathrm{Y}}^{2}=\sigma_{\mathrm{Z}}^{2}$ and an equal number of controls and cases in each cluster it follows that

$$
\operatorname{Var}\left(\bar{y}_{i j}-\bar{z}_{i j}\right)=2 *(1-\rho) \sigma^{2} / n_{i j} .
$$

Then the variance of the estimate of $Q$ turns out to be

$$
\operatorname{Var}(Q)=\frac{2 \sigma^{2}}{\left(\Sigma w_{i j} n_{i j}\right)^{2}} \Sigma w_{i j}^{2} n_{i j}[1+(b-2) \rho]
$$

where

$$
b=\frac{\sum w_{i j} n^{2}}{\sum w_{i j} n}
$$


The design effect is the ratio of (4) and the variance under simple random sampling. Under simple random sampling the variance is $\operatorname{Var}\left(Q^{\prime}\right)=2 \sigma^{2} / N$. The design effect is computed as

$$
D E=\frac{N \sum_{i j} w_{i j}^{2} n_{i j}}{\left(\sum_{i j} w_{i j} n_{i j}\right)^{2}} \bullet[1+(b-2) \rho] .
$$

where $b$ is defined as before. The design effect can be split into the probability design effect (first term of the product in (5) ) and the cluster design effect (second term of (5)). 


\section{References}

(1) Anderson, R., Kasper, J., Frankel, M. and Associates, Total survey error, Jossey-Bass Publishers, San Francisco, 1979.

(2) Cochran, W.G., Sampling techniques, John Wiley \& Sons, New York, 3rd ed, 1997.

(3) Frankel, M.R., Shapiro, M.F., Duan, N., Morton, S.C., Berry, S.H., Brown, J.A., Burnam, M.A., Cohn, S.E., Goldman, D.P., McCaffrey, D.F., Smith, S.M., St Clair, P.A., Tebow, J.F. and Bozzette, S.A.,"National probability samples in studies of low-prevalence diseases. Part II: designing and implementing the HIV cost and services utilization study sample," Health Services Research, 34, 5, 969-992, 1999.

(4) Gabler, S., Haeder, S. and Lahiri, P., “A model based Justification of Kish's formula for design effects for weighting and clustering,” Survey Methodology, 25, 1, 105-106, 1999.

(5) Goldman D., Adams, J.L., Berry, S.H., Escarce, J.J., Kilgore, M., Lewis, J., Rosen, M.R., Schoenbaum, M.L., Schonlau, M., Wagle, N. and Weidmer, B.A., "Measuring the incremental costs of clinical cancer research: the cost of cancer treatment study,” RAND, Santa Monica, CA, report MR-1169-NSF, 2000.

(6) Kish, L., Survey sampling, John Wiley \& Sons, New York, 1965.

(7) Linacre, S., Trewin, D., "Total survey design - application to a collection of the construction industry,” Journal of Official Statistic, 9, 3, 611-621, 1993.

(8) Mansour, E.G., "Barriers to clinical trials. Part III: knowledge and attitudes of health care providers,” Cancer, 74, (suppl 9), 2672-2675, 1994.

(9) Morrow, G.R., Hickok, J.T. and Burish, T.G., "Behavioral aspects of clinical trials. An integrated framework from behavior theory,” Cancer, 74, (suppl 9), 2676-2682, 1994.

(10) Shapiro, M.F., Berk, M.L., Berry, S.H., Emmons, C.A., Athey, L.A., Hsia, D.C., Leibowitz, A.A., Maida, C.A., Marcus, M., Perlman, J.F., Schur, C.L., Schuster, M.A., Senterfitt, J.W. and Bozzette, S.A., "National probability samples in studies of lowprevalence diseases. Part I: perspectives and lessons from the HIV cost and services utilizations study,” Health Services Research, 34, 5, 951-948, 1999.

(11) Wagner, J.L., Alberts, S.R., Sloan, J.A., Cha, S., Killian, J., O’Connell, M.J., Van Grevenhof, P., Lindman, J. and Chute, C.G., "Incremental costs of enrolling cancer patients in clinical trials: a population-based study," Journal of the National Cancer Institute, 91, 10, 847-853, 1999. 
Figure 1: Summary of the 4 steps for picking a sampling design. The gray shaded steps apply to each individual design separately.

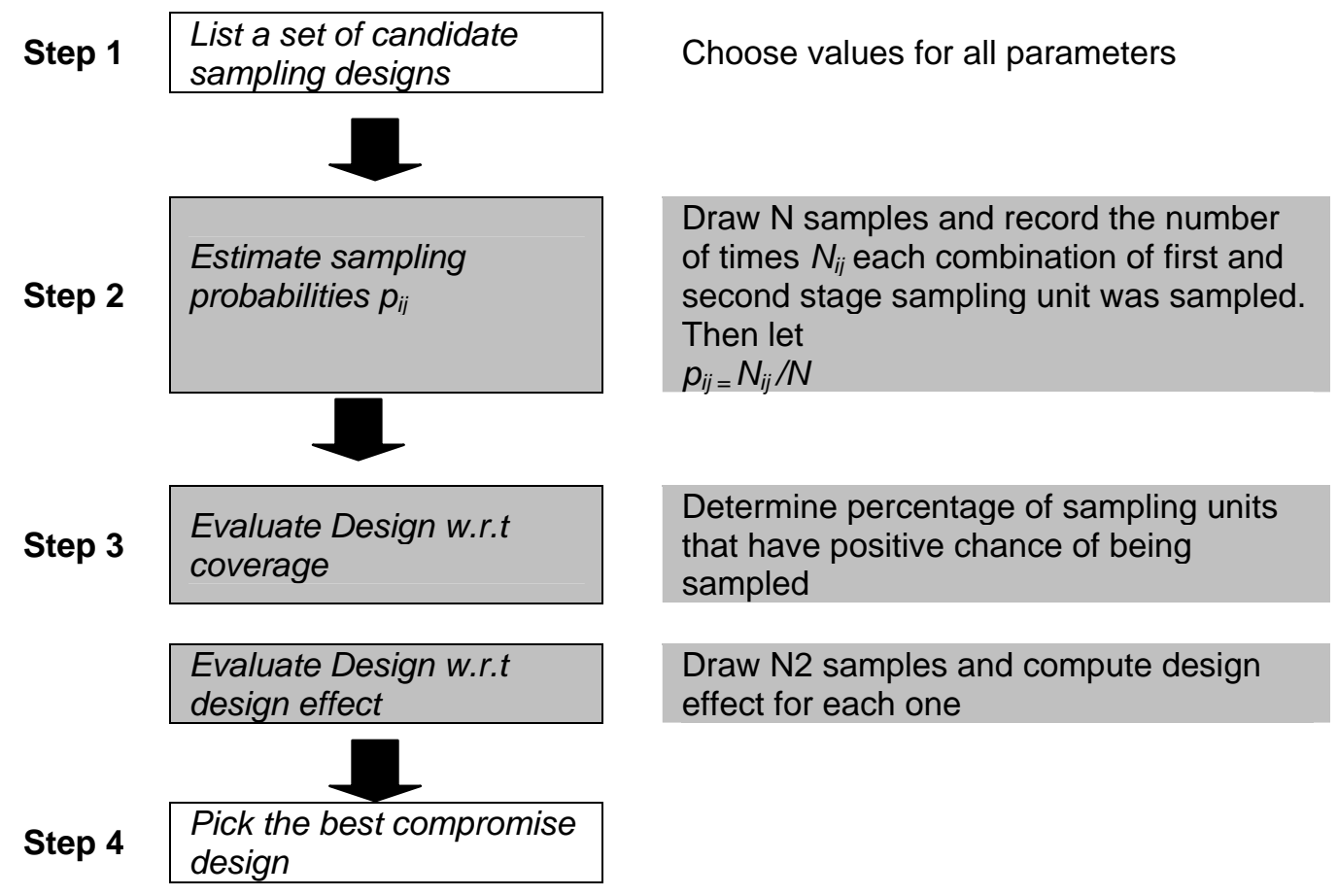


Figure 2. Histogram of the number of patients accrued in our clinical trials in a 6 months window. The horizontal axis refers to the size of the trials, the vertical axis to the total number of patients accumulated in these trials. For example, trials with 16-31 patients accrued together accounted for a total of about 350 patients.

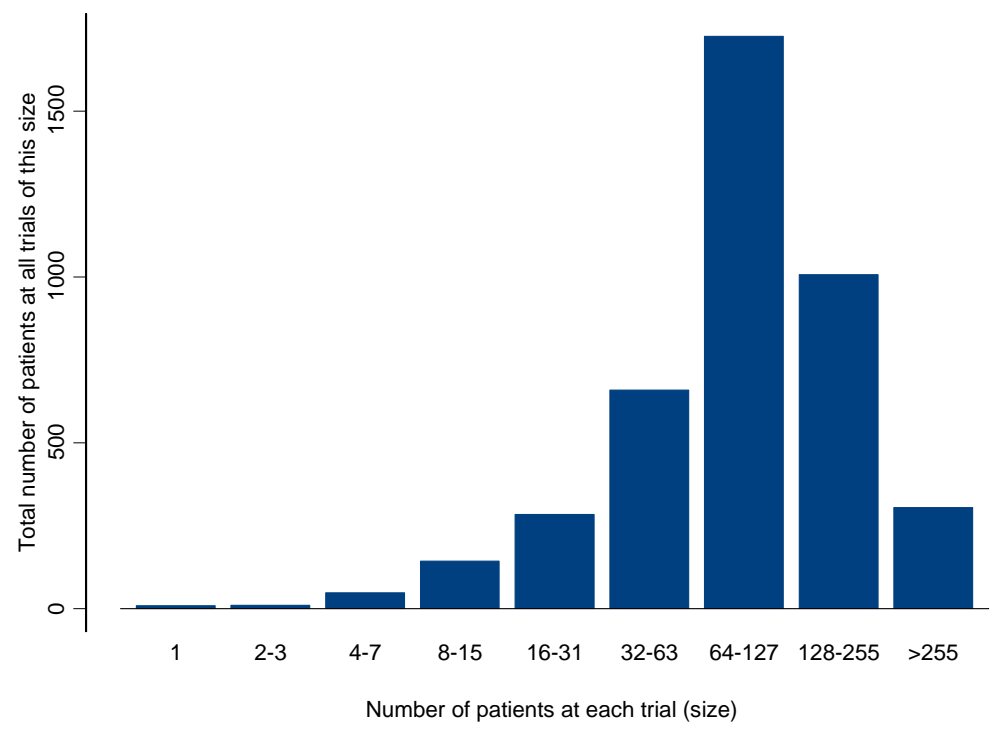

Figure 3. Histogram of the number of patients accrued in institutions in a 6 months window. The horizontal axis refers to the size of institutions, the vertical axis to the total number of patients accumulated in these institutions. For example, institutions with 9-12 patients accrued accounted for a total of about 600 patients.

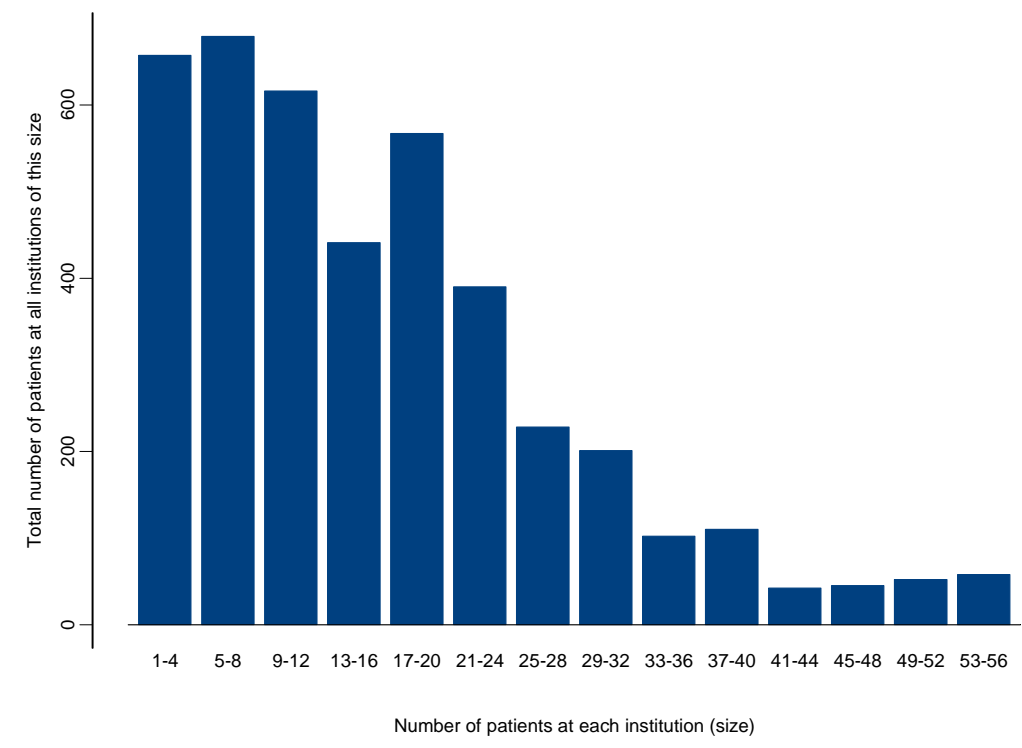


Figure 4: Simulation of 3 scenarios: (1) 50 Trials, 50 Institutions; (2) 35 Trials, 55 Institutions; (3) 45 Trials, 50 Institutions. For each scenario we explore all combinations of 3 levels each for the institution cutoff, trial cutoff and $\alpha$. The effective sample size and sample frame coverage for each of these runs are displayed. Efficient frontiers are drawn for the three scenarios. Sample frame coverage is measured relative to the size of the sample frame.

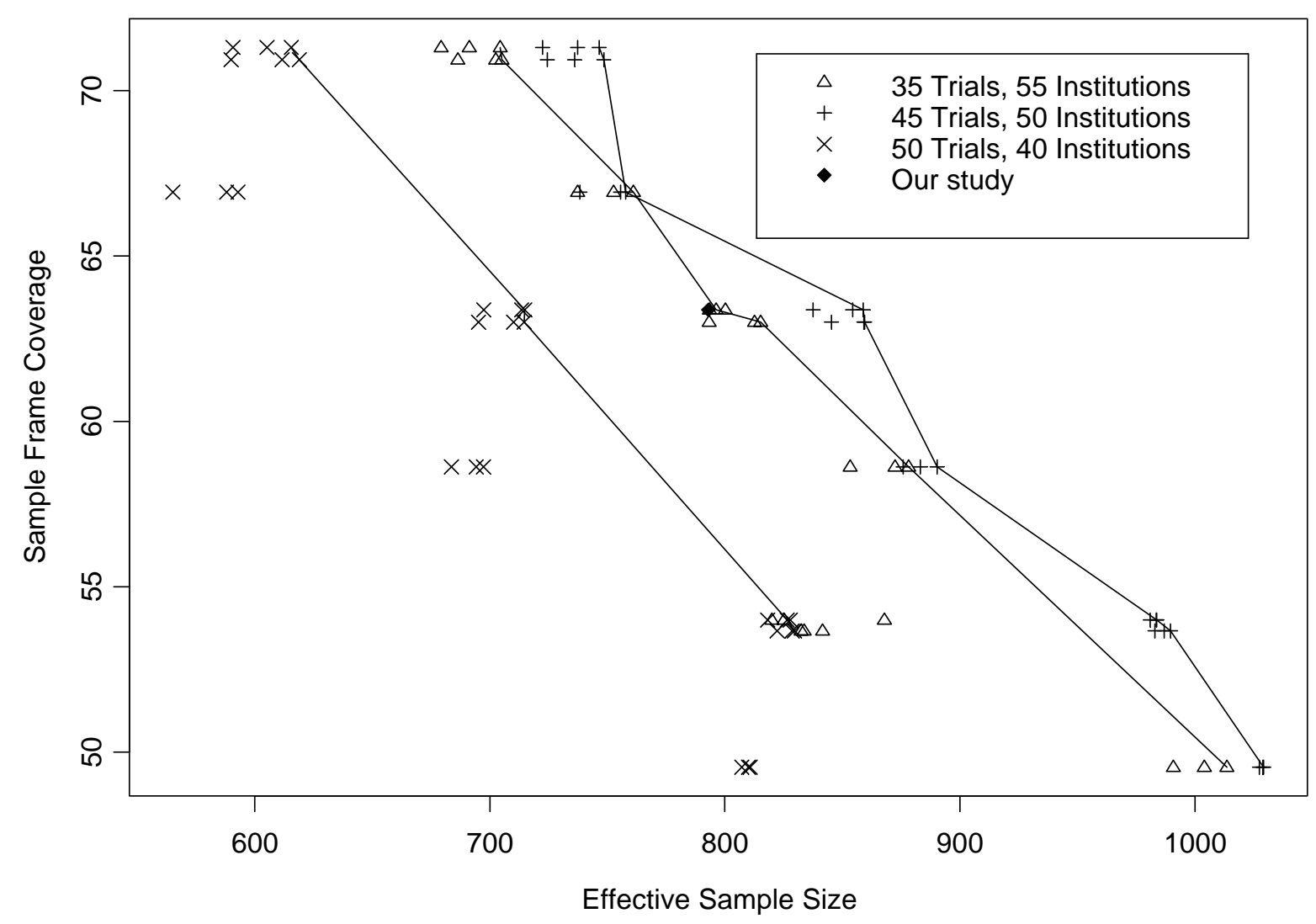


Table 1: Various statistics for all 27 runs for the scenario with 35 trials and 55 institutions. Sample frame coverage is given in percent relative to a sample frame with trial cutoff and institution cutoff equals to zero $(=100 \%)$.

\begin{tabular}{|c|c|c|c|c|c|c|c|c|c|}
\hline $\begin{array}{l}\text { Trial } \\
\text { Cutoff }\end{array}$ & $\begin{array}{l}\text { Insti- } \\
\text { tution } \\
\text { Cutoff }\end{array}$ & Alpha & Accrual & $\begin{array}{r}\text { Effective } \\
\mathrm{N}\end{array}$ & $\begin{array}{r}\text { Coverage } \\
\text { (\%) }\end{array}$ & $\begin{array}{r}\text { Median } \\
\text { Design } \\
\text { Effect }\end{array}$ & $\begin{array}{c}\text { 90th } \\
\text { Percentile } \\
\text { Design } \\
\text { Effect C }\end{array}$ & $\begin{array}{r}\text { Median } \\
\text { luster DE }\end{array}$ & $\begin{array}{r}\text { Median } \\
\text { Probability } \\
\text { DE }\end{array}$ \\
\hline 18 & 6 & 0.5 & 939 & 704 & 71.3 & 1.32 & 1.54 & 1.02 & 1.29 \\
\hline 24 & 6 & 0.5 & 933 & 705 & 70.9 & 1.32 & 1.53 & 1.02 & 1.29 \\
\hline 30 & 6 & 0.5 & 967 & 761 & 66.9 & 1.26 & 1.33 & 1.02 & 1.23 \\
\hline 18 & 9 & 0.5 & 1047 & 800 & 63.4 & 1.29 & 2.25 & 1.02 & 1.26 \\
\hline 24 & 9 & 0.5 & 1049 & 813 & 63.0 & 1.27 & 2.38 & 1.02 & 1.24 \\
\hline 30 & 9 & 0.5 & 1079 & 878 & 58.6 & 1.22 & 1.38 & 1.03 & 1.19 \\
\hline 18 & 12 & 0.5 & 1151 & 868 & 54.0 & 1.31 & 2.23 & 1.03 & 1.28 \\
\hline 24 & 12 & 0.5 & 1156 & 832 & 53.7 & 1.37 & 2.16 & 1.03 & 1.34 \\
\hline 30 & 12 & 0.5 & 1188 & 991 & 49.5 & 1.20 & 1.45 & 1.04 & 1.16 \\
\hline 18 & 6 & 0.75 & 977.5 & 691 & 71.3 & 1.40 & 1.66 & 1.02 & 1.37 \\
\hline 24 & 6 & 0.75 & 985 & 702 & 70.9 & 1.39 & 1.64 & 1.02 & 1.37 \\
\hline 30 & 6 & 0.75 & 1003 & 753 & 66.9 & 1.33 & 1.40 & 1.02 & 1.30 \\
\hline 18 & 9 & 0.75 & 1078 & 796 & 63.4 & 1.34 & 1.97 & 1.02 & 1.31 \\
\hline 24 & 9 & 0.75 & 1081 & 815 & 63.0 & 1.32 & 2.29 & 1.02 & 1.29 \\
\hline 30 & 9 & 0.75 & 1109 & 872 & 58.6 & 1.27 & 1.46 & 1.02 & 1.23 \\
\hline 18 & 12 & 0.75 & 1179 & 825 & 54.0 & 1.42 & 2.10 & 1.02 & 1.40 \\
\hline 24 & 12 & 0.75 & 1181 & 842 & 53.7 & 1.40 & 2.06 & 1.03 & 1.37 \\
\hline 30 & 12 & 0.75 & 1208.5 & 1004 & 49.5 & 1.20 & 1.61 & 1.04 & 1.16 \\
\hline 18 & 6 & 1 & 1023.5 & 679 & 71.3 & 1.49 & 1.88 & 1.01 & 1.47 \\
\hline 24 & 6 & 1 & 1025 & 686 & 70.9 & 1.48 & 1.83 & 1.01 & 1.46 \\
\hline 30 & 6 & 1 & 1048 & 737 & 66.9 & 1.41 & 1.53 & 1.02 & 1.38 \\
\hline 18 & 9 & 1 & 1112 & 793 & 63.4 & 1.39 & 2.26 & 1.02 & 1.36 \\
\hline 24 & 9 & 1 & 1116 & 793 & 63.0 & 1.39 & 2.62 & 1.02 & 1.37 \\
\hline 30 & 9 & 1 & 1133 & 853 & 58.6 & 1.32 & 1.57 & 1.02 & 1.29 \\
\hline 18 & 12 & 1 & 1201.5 & 820 & 54.0 & 1.47 & 2.13 & 1.02 & 1.43 \\
\hline 24 & 12 & 1 & 1209 & 834 & 53.7 & 1.43 & 2.32 & 1.02 & 1.39 \\
\hline 30 & 12 & 1 & 1229 & 1013 & 49.5 & 1.21 & 1.46 & 1.04 & 1.17 \\
\hline
\end{tabular}




\section{Affiliation of Authors}

John L. Adams Ph.D., RAND, john_adams@rand.org

Matthias Schonlau Ph.D., RAND, matthias_schonlau@rand.org

José J. Escarce M.D., RAND, jose_escarce@rand.org

Meredith Kilgore M.S.P.H., University of Alabama, mkilgore@ms.soph.uab.edu

Michael Schoenbaum Ph.D., RAND, michael_schoenbaum@rand.org

Dana P. Goldman Ph.D., RAND, dana_goldman@rand.org

Corresponding Author: Matthias Schonlau

Office: RAND, Statistics Group, P.O. Box 2138, Santa Monica, CA, 90407-2138

Phone: 310-393-0411 x7682, email: matthias_schonlau@rand.org

\section{Acknowledgement:}

The National Cancer Institute is providing principal funding for the Cost of Cancer Treatment Study. Additional funding comes from the Office of the Director, National Institutes of Health and by the National Science Foundation as part of its support for the White House's Office of Science and Technology Policy. The authors wish to thank Sandra Berry, Ronald Fricker, Richard Kaplan, Daniel McCaffrey, Michael Montello, Sally Morton, Mary McCabe, Arnold Potosky, and Jane Weeks for their assistance in this research. 